\title{
AN INTRODUCTION TO MORPHOLOGY OF THE REPRODUCTIVE SYSTEM AND ANATOMY OF HEN'S EGG
}

\author{
Md. Anisur Rahman \\ Department of Zoology, University of Rajshahi, Rajshahi-6205, Bangladesh \\ e-mail: ar_zool.uk@fastermail.com
}

\begin{abstract}
The present study was designed to investigate the morphology of reproductive system and egg anatomy of the domestic hen (Gallus domesticus L.). The system consists of oviduct and ovary. The oviduct consists of infundibulum, magnum, isthmus, uterus and vagina which are sole distributor for making nutrition enriched egg. The anatomy of egg revealed that there are calcareous eggshell, shell membranes, egg white, vitelline membrane, egg yolk, and germinal disc. The fertilized egg showed a concentric circle around the nucleus known as blastoderm that contained area pellucida and area opaca whereas an unfertilized egg showed nucleus as white spot (blastodisc). Primordial germ cells (PGCs) are progenitor cells of ova and spermatozoa and they originate from the epiblast of the central part of the area pellucida. The microscopic structure of eggshell rendered leathery cuticle, fibrous matrix and shell membranes. The egg protects itself by its own mechanism from being injured and provides a complete diet for the developing embryo.
\end{abstract}

Key words: Ovary, oviduct, egg, blastodisc, eggshell

সারাংশ: বর্তমান অধ্যয়নটি মুরগির (Gallus domesticus L.) প্রজননতন্ত্রের অঙসংস্থান এবং ডিনের অঙ্গব্যবচ্ছেদ অনুসন্ধান করার জন্য আলেখন করা হয়েছে। প্রজননতন্ত্রটিতে ডিম্বনালী এবং ডিম্বাশয় থাকে। ডিম্বনালীতে Infundibulum, Magnum, Isthmus, Uterus এবং Vagina অংশ থাকে যারা পুষ্টিসমৃদ্ধ ডিম টৈরিতে একক ভূমিকা পালন করে। ডিনের অঙ্যবচ্ছেদে জানা যায় যে, ক্যালসিয়াম সমৃদ্ধ ডিম্বখোলক, খোলকপর্দা, ডিনের সাদা অংশ, ডিম্বকপর্দা, ডিম্বকুসুম এবং জার্মিনাল চাকতি ডিনে বিদ্যমান। নিষিক্ত ডিম্বাণুর নিউক্লিয়ালের চারপাশে একটি এককেন্দ্রীক বৃত্ত থাকে যেখানে Area pellucida এবং Area opaca বিদ্যমান এবং একে ব্রাস্টোডার্ম বলে। পক্ষান্তরে, অনিষিক্ত ডিম্বাণুর নিউক্লিয়াসটি সাদা ফোঁটা দাগ হিসাবে থাকে এবং একে র্রাল্টোডিস্ক বলে। আদি অংকুর কোষ (PGCs) ক্তক্রাণু এবং ডিম্বাণুর পূর্বসূরী কোষ যারা Area pellucida-র কেন্দ্রীয় অংশ Epiblast থেকে উৎপত্তি লাভ করে। ডিম্বখোলকের আণুবীক্ষণিক গঠনে চর্মসদৃশ কিউটিকল, তন্তময় ম্যাট্রিক্স এবং খোলকপর্দা দেখা যায়। ডিম নিজস্ব উপায়ের মাধ্যনে আঘাতপ্রাপ্ত হওয়া থেকে নিজেকে রক্ষা করে এবং বর্ধনশীল ভ্রংণের পূর্ণাঙ্গ খাদ্য সরবরাহ করে।

\section{Introduction}

The domestic hen provides a unique model for the study of the reproductive system and the mechanisms underlying the egg production. The current poultry farming is established with the domesticated fowl used for both meat and egg production. This farming also includes birds like chicken, turkey, duck, goose, ostrich, quail, pheasant, guinea fowl and peafowl (Rahman 2009). Chickens are the most popular poultry worldwide irrespective of culture and religion (Roenigk 1999; Aho 2001), because of high nutritive values of poultry products. Chicken meat and eggs are the major protein source for consumers in most of the countries around the world, and poultry contributes about 22 to 27 percent of the total animal protein supply in Bangladesh (Ahmed \& Haque 1990). Poultry is a promising sector for poverty reduction in Bangladesh.

In the context of Bangladesh, deshi (local) chickens are reared in the villages mainly by the women whereas the exotic chickens are used as farm animals reared both in villages and urban areas. The exotic chickens are commercially reared because they are high yielding (eggs and meat) than the indigenous breeds (Hossain 1992). It is often claimed that the reproductive performances of the native chickens are very poor (Okada et al. 1988) in comparison to different exotic pure breeds and hybrids.
Poultry production and poultry related industry contributes most significantly to the total livestock sector in Bangladesh. Poultry is in the top position of the livestock sector, contributing $21 \%$ of the total livestock contribution (Khan and Roy 2003), 20\% of the protein consumed originates from poultry $(90 \%$ chicken followed by ducks $8 \%$ and a small number of quail, pigeons and geese) (Das et al. 2008). It is estimated that there are about 140 million chickens and 13 million ducks in the total poultry population (DLS 2000) are scattered throughout rural areas in Bangladesh.

The highly nutritious egg is a female sex cell produced in the ovary and either fertilization occurred in the infundibulum or not subsequently achieves its mature structure after a long journey through reproductive tract or oviduct. The oviduct is a useful model for hen reproductive biology. Active ovary and oviduct have well enough secretory functions and it ensures quality egg. Any abnormality in the reproductive tract or egg materials may reduce the egg laying frequency as well as egg quality and it could be an indication on the process of quality egg laying. Thus, it could be important for the poultry farmers and the poultry researchers. 
Number of works on the morphological studies of the ovary and oviduct and the anatomy of egg of chicken and other poultry species have been done but a detailed study has not yet been published on the hen Gallus domesticus. The purpose of the study therefore was to investigate the different morphological characteristics of the reproductive system and the anatomical parameters of eggs of high egg producing Rhode Island Red (RIR) hen.

\section{Materials and Methods}

The study was conducted at Genetics and Molecular Biology Laboratory, Department of Zoology, University of Rajshahi, Bangladesh. The materials used for the study were rearing cages $(50 \mathrm{~cm} \mathrm{X} 36 \mathrm{~cm} \mathrm{X} 40$ $\mathrm{cm} ; 2$ in number), food and water pot, electronic balance, microscope, dissecting box, petri dishes (19.5 $\mathrm{cm}, 11.5 \mathrm{~cm}$ and $9 \mathrm{~cm}$ diameter), conical flask (500 ml), measuring scale and a digital camera (Canon IXY).

Experimental bird: The experimental birds were two in number and they were RIR (Rhode Island Red) hen collected from local poultry farm of Rajshahi City Corporation. The individual hen was 42 weeks of age and weight was around $2.45 \mathrm{~kg}$ at the beginning of the experiment.

Rearing of the bird: The laying birds were reared in cases made of wood for 21 days and provided supplemented feed during the day time. The rate of food supply was $125 \mathrm{~g}$ layer feed (commercial) and 25 g rice per day while $100 \mathrm{ml}$ drinking water was supplied every day individually. The hens were kept in the laboratory illuminated for 14 hour per day at room temperature $\left(30 \pm 2^{\circ} \mathrm{C}\right)$ and at $80 \pm 5 \% \mathrm{RH}$.

Collection of eggs: The eggs (40 in number) were collected every day in the morning, or noon or evening whenever the hen laid and kept in the rearing room with identical conditions. Fertilized eggs (within one month after oviposition; 10 in number) were collected from local market of Rajshahi City Corporation because the experimental layer birds laid only unfertilized egg.

Preparation of reagents: Saline water was prepared by $\mathrm{NaCl}(0.87 \%$; w/v). Initially $4.35 \mathrm{~g} \mathrm{NaCl}$ was added in $500 \mathrm{ml}$ distilled water in a conical flask $(500 \mathrm{ml})$. The reagents needed in the experiment were also prepared. Ethylenediamine tetraacetic acid (200 mM EDTA) solution was prepared by adding 37.224 g EDTA into $500 \mathrm{ml}$ distilled water. EDTA solution or chelating solution was used to dissolve the eggs shell.
Removal of internal organs: The hens were dissected from neck to anus to observe the various internal organs. Ovary and oviduct were removed and kept in $\mathrm{NaCl}$ solution $(0.87 \%)$ for further processing. The replications for each reproductive organ were two in number and the best one is represented as figure for each and described accordingly in the text.

Dissection of egg: Freshly laid eggs were washed with distilled water and kept on the table for drying. 10 eggs were broken with the light touch of scissors or scalpel and then some 20 eggs were cut in equatorial region and kept in $0.87 \% \mathrm{NaCl}$ solution to observe their constituent parts. Some 20 eggs (unfertilized 10 and fertilized 10) were decanted and used for the study of yolk with blastodisc/ blastoderm. The vitelline membranes were separated from the egg yolk manually. The constituent parts of eggs were observed and the best one of each has been presented in the text as replication representative.

Collection of egg shells: Egg shells were collected from the eggs by decanting the egg yolk and albumin. The empty shells were washed well so that no albumin remains attached to them and then dried for few days at room temperature in the laboratory.

Collection of cuticle, shell matrix and shell membranes: The dried eggshells were broken into several pieces and kept in petri dishes containing 200 mM EDTA solution for dissolving the eggshells. The calcareous substance of eggshell was removed by EDTA within 3-4 days. The isolated envelopes were cut into small pieces, washed three times with saline water and used for microtomy.

Light microscopy: The constituent parts of eggs were kept in saline water $(0.87 \%)$ in petri dishes and observed under a light microscope. Special attention was paid to observe the nucleus of fertilized and unfertilized eggs of hen.

Histology: The decalcified shell membranes were fixed in Bouin's solution and embedded in paraffin wax and cut into thin section $(5 \mu \mathrm{m})$. The sections were processed and mounted permanently by canada balsum.

Photography: Photographs of reproductive system and egg of hen were taken by using a digital camera (Model: Cannon IXY, Japan). 


\section{Results \\ Morphology of the female reproductive system}

The female reproductive system of the chicken is divided into two separate parts: the ovary and the oviduct. In the majority of avian species including chicken only the left ovary and oviduct are functional. Although the right one is present embryologically, it regresses during development and is vestigial in the adult bird. The egg is produced in the ovary as follicles and after maturation they are engulfed by funnel of the infundibulum. Either fertilization occurs in the infundibulum or not, the egg passes a long path through the oviduct and resides in the uterus for 5-25 hour (Woodward and Mather 1964; Rahman et al. 2009a, Iwasawa et al. 2010). The egg then expelled out from the reproductive tract with facing either its sharp end or blunt end to the exterior. Just after laying the egg is soft and gradually the outermost covering/shell becomes hardened structure in presence of air.

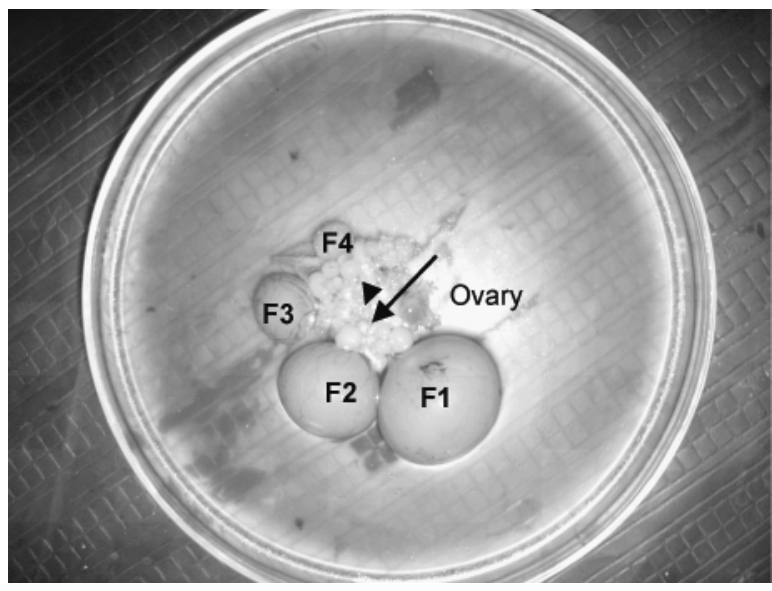

Fig. 1 Photograph showing ovary with cluster of mature and immature follicles. Mature follicles are indicated by F1, F2, F3 and F4. Arrow shows immature or growing follicles (small and yellow) and arrowhead indicates primary or empty follicles (small and transparent).

The mature ovary has a grapelike cluster of developing yolks or ova of which some are small, some are large and prominent and they are identified easily. The left ovary is found in the anterior body cavity left to the kidney and adjacent to the adrenal glands. It is attached to the body wall by a thin ligamentous structure called the mesovarian. A large number of follicles are found in the ovary which become matured serially (Fig. 1). The matured eggs or follicles are grasped later by the funnel of the infundibulum. As the breeding season approaches, several follicles enter into a phase of rapid growth and maturation, accumulate yolk just before ovulation.

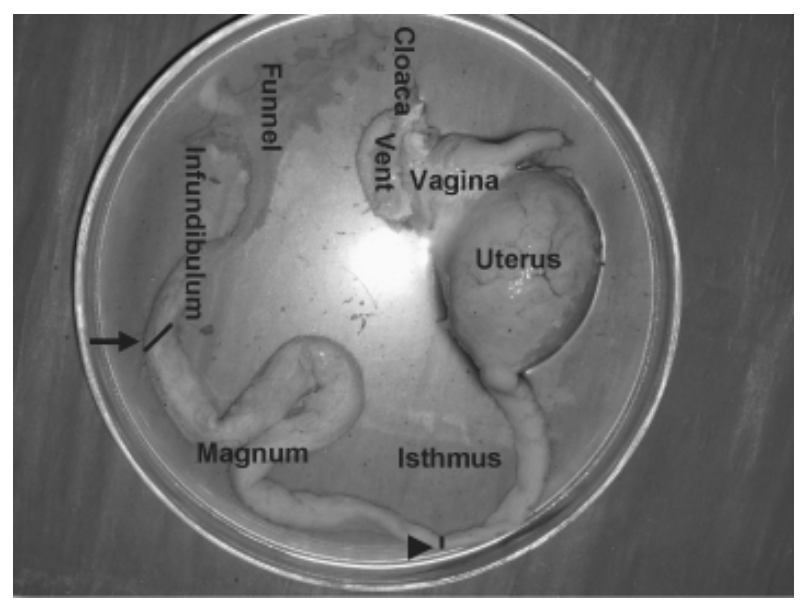

A

Ovary

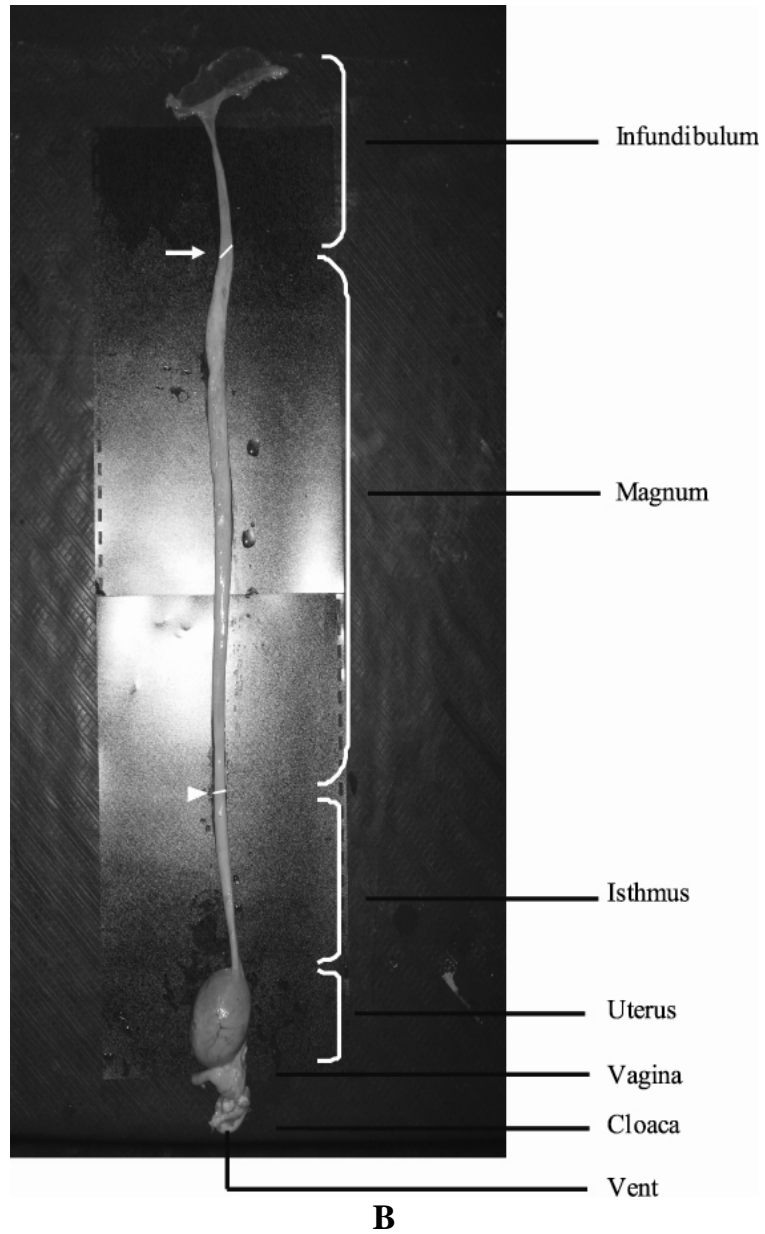

Fig. 2 Oviduct of the RIR hen showing infundibulum $(10 \mathrm{~cm})$, magnum $(33 \mathrm{~cm})$, isthmus $(15 \mathrm{~cm})$, uterus $(7 \mathrm{~cm})$, vagina $(3 \mathrm{~cm})$ and cloaca. A, reproductive tract in a compact condition and B, reproductive tract in an expanded condition. Arrow indicates infundibulum-magnum junction and arrowhead shows magnumisthmus junction. 


\section{Oviduct}

The second major part of the hen's reproductive system is the oviduct (Fig. 2. A \& B), which was found as $68 \mathrm{~cm}$ in length. The oviduct is attached to the dorsal body wall by the mesotubarium ligament. In the immature or nonbreeding hen, the oviduct is an inconspicuous, narrow tube, straight and uniform in diameter. In the reproductively active female bird, the oviduct undergoes tremendous enlargement and occupies a large part of the abdominal cavity. The increase in length causes a folding of the oviduct upon itself. Oviduct is divided into five distinct regions associated with egg formation: (1) infundibulum, (2) magnum, (3) isthmus, (4) uterus or shell gland, and (5) vagina.

Infundibulum: The first section of the oviduct is infundibulum, $10 \mathrm{~cm}$ in length and has two portions; a thin walled funnel which opens into a thick walled tubular region which constitutes the second portion of the infundibulum. The funnel opening is an elongated slit which faces towards the ovary. The funnel is brownish or whitish in colour, the next portion is reddish due to the presence of tubular glands and enriched vascularization. The infundibulum and magnum portions are separated from each other by leaving a demarcation region seems to be an oblique line (Fig. 2. arrow) after which comparatively less reddish magnum starts.

Magnum: The magnum, $33 \mathrm{~cm}$ long and is the largest section of the oviduct. It is readily distinguished from the infundibulum by its coiling nature, greater external diameter and markedly thicker wall caused by the presence of numerous glands packed into massive mucosal folds.

Isthmus: The third section of the oviduct, the isthmus is $15 \mathrm{~cm}$ long. The isthmus, as its name implies, is slightly constricted ('isthmus' refers to a narrow band of tissue connecting two larger parts of an anatomical structure), and has two parts i.e., upper white isthmus and lower red isthmus from where the inner and outer shell membranes are added, respectively. The constriction separates the magnum and isthmus is marked by a thin, translucent line (Fig. 2. arrowhead) which can be seen on the mucosal surface with the
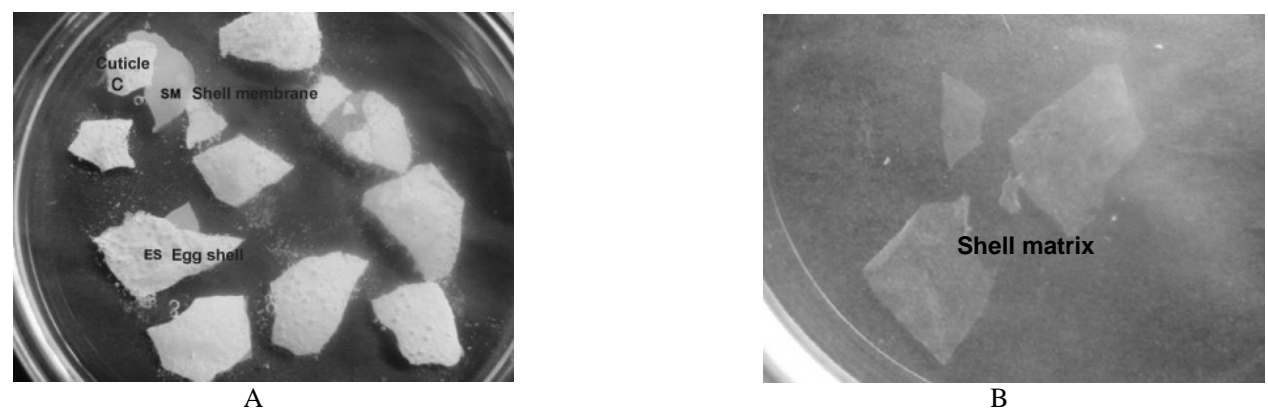

Fig. 3 Figures show eggshell parts after dissolving calcareous substances. A dissolving state (by EDTA) of egg shell showing cuticle (C), shell membrane (SM) and egg shell (ES; calcareous part). B decalcified shell matrix (without cuticle and shell membrane).

unaided eye. The segment of the oviduct immediately succeeding the isthmus is of similar diameter but after a short course, expands to form a uterus in which the egg is retained during the entire period of shell formation.

Shell gland or uterus: The next section of the oviduct is the shell gland or uterus. The shell gland is $7 \mathrm{~cm}$ long, reddish in colour and pouch like in structure. The pouch always expands and contracts for which when egg comes in the uterus, the egg rotates. The egg remains in uterus for 19-20 hours of laying cycle.

Vagina: The vagina is the short and terminal portion of the oviduct measuring $3 \mathrm{~cm}$ long proximal to its opening into the urodeum. This part does not really play role in egg formation but is important in laying of the egg. Powerful muscles of the vaginal wall and a welldeveloped muscular sphincter at the uterine-vaginal junction serve to expel the egg out of the hen's body.

Cloaca: The cloaca is the terminal chamber of the gastrointestinal and urogenital systems, opening at the vent. The vent in chicken is the common opening of cloaca through which intestinal, urinary and reproductive tracts become empty.

\section{Anatomy of egg}

Chicken has highly complex reproductive cell (the ovum), which is essentially a tiny center for life. Development of the embryo initially takes place in the blastoderm in case of fertile egg. The albumen enclosed by the shell membranes surrounds the egg yolk and protects this potential life. It is an elastic, shockabsorbing viscous material with higher percentage of water content. In case of chicken the yolk and albumen are prepared to support the life of a growing embryo for three weeks. This entire mass is surrounded by two shell membranes (internally) and an external hard covering called the shell. The shell provides for an exchange of gases and mechanical (physical) as well as physiological (antimicrobial) means of conserving the food and water supply within the egg. Except the blastodisc, egg-yolk and inner vitelline membrane, all parts of the egg are formed in the oviduct. An egg is a single cell until it is fertilized. A chicken egg has the following anatomical structures: 


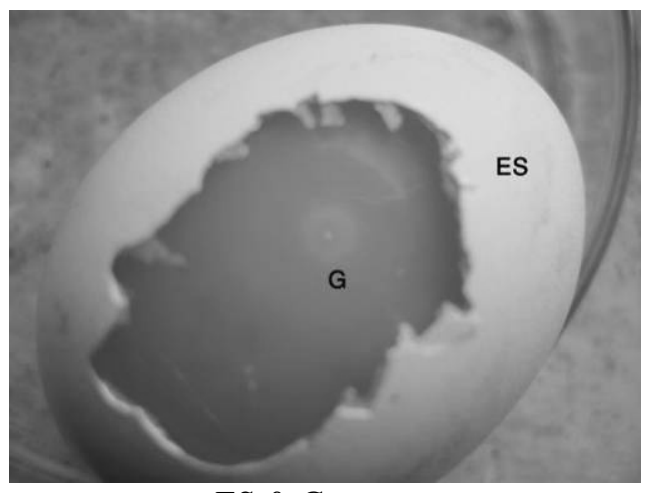

ES \& G

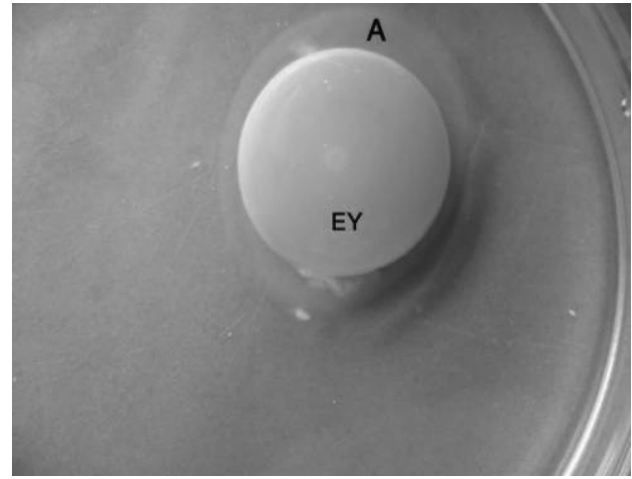

A \& EY

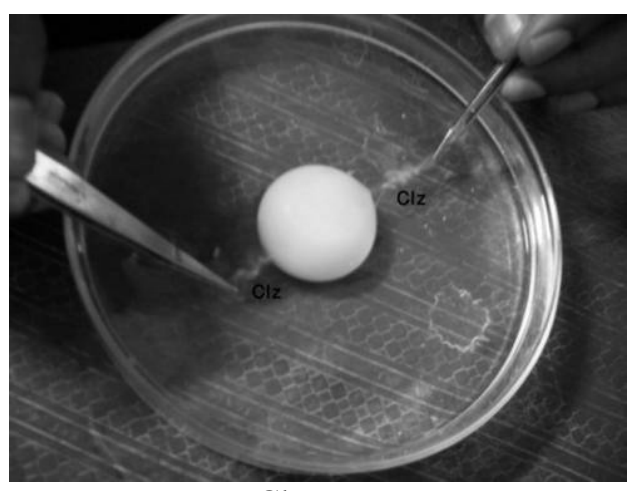

$\mathrm{Clz}$

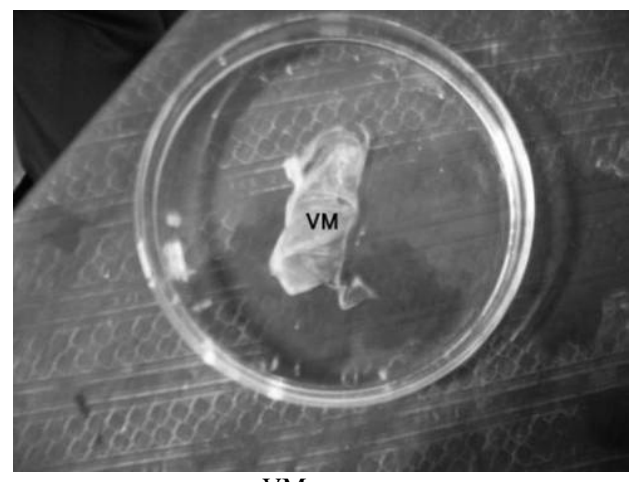

VM

Fig. 4 Different parts of an egg. ES-Egg shell; G- Germinal disc; Clz-Chalaza; A-Albumen/egg white; EY-Egg yolk; VM-Vitelline membrane.

Egg shell: The hard outer surface of egg is the shell (Fig. 3; ES). It provides protection to the inner portion of the egg and to the structure of embryo during incubation. The eggshell is composed of approximately 95\% calcium carbonate crystals, which are stabilized by protein matrix. Normally, the shape of egg is oval but sometimes might be different. Microscopic studies show that the hen's egg shell is a highly ordered structure throughout its entire thickness. The eggshell is composed with three parts, cuticle (Fig. 3 A. C), matrix (Fig. 3 B.) and shell membrane (Fig. 3 A; SM). The cuticle is brownish as it contains the pigment protoporphyrin and the matrix is whitish (Fig. 3. B), located between the cuticle and shell membrane.

Shell matrix: After decalcification of eggshell there is a semi transparent proteineous vesicular structure (Fig. 3. B) between the cuticle and shell membranes, which is called egg shell matrix. Layers of vesicles were interconnected with each other by fibers or fiber sheets and they all are hidden by different types of calcites during eggshell formation in the uterus (Hernández et al. 2008; Hincke et al. 2008).

Shell membranes: The shell membranes lie between the eggshell and the egg white (Fig. 3. A; SM). Two white proteinous membranes termed as shell membranes, protect the embryo and all the inner structures supporting it. Microscopic view (Fig. 5) apparently shows the two membranes differ in their structure: smooth one is towards the egg white (inner) and comparatively rough layer is towards the egg shell.

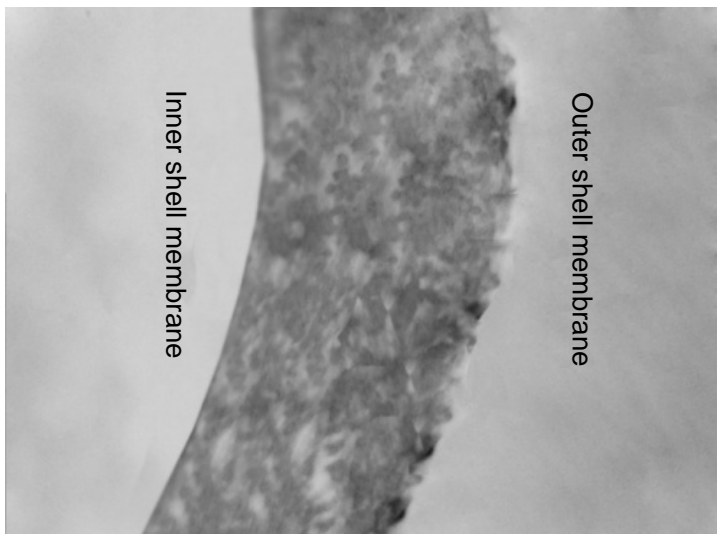

Fig. 5 Microscopic view of decalcified shell membranes showing the inner smooth and outer rough edges.

Air cell: The air cell is the space between the outer and inner shell membranes at the egg's blunt end, where the shell is most porous, and air can easily enter. When the egg is laid the inner portion of the egg contracts and 
forms an air cell between the two shell membranes, and gradually expands as the embryo matures. It is also found in hard cooked egg.

Chalazae: Inside the egg, yolk is suspended in the egg white, hanged by two spiral bands of tissue called chalazae. The chalazae hold the yolk securely in the center of the egg, while allowing play to absorb shock. These are twisted ropes of protein fibers attached with vitelline membrane of egg inside and with egg shell outside (Burley and Vadehra 1989) (Fig. 4. Clz ).

Vitelline membrane: The casing that enclose the yolk is called as vitelline membranes (Fig. 4. VM). This membrane has inner and outer layers. The inner layer is transparent, encircles the blastodisc with yolk. The outer layer is whitish, has been described as inner portion of chalaziferous layer (Rahman et al. 2007, 2009b) rather than outer vitelline membrane (Back et al. 1982).

Germinal disc: On the yolk surface, there is a germinal disc (Fig. 4. G) enclosed by inner vitelline membrane. This is a small disc of cytoplasm containing the DNA molecules of the ovum. The germinal disc or blastodisc is visible with the naked eye, appearing on the surface of the yolk as a white dot. In case of fertile egg, the germinal disc is called blastoderm which has a concentric circle around the nucleus and there is centrally a transparent zone called area pellucida and peripherally non transparent zone the area opaca (Fig. 6 . A). The primordial germ cells (PGCs) originate from the epiblast, the central part of the area pellucida which are the progenitor cells of ova and spermatozoa of adult. The infertile egg, there is only a dot like structure called blastodisc (Fig. 6. B).

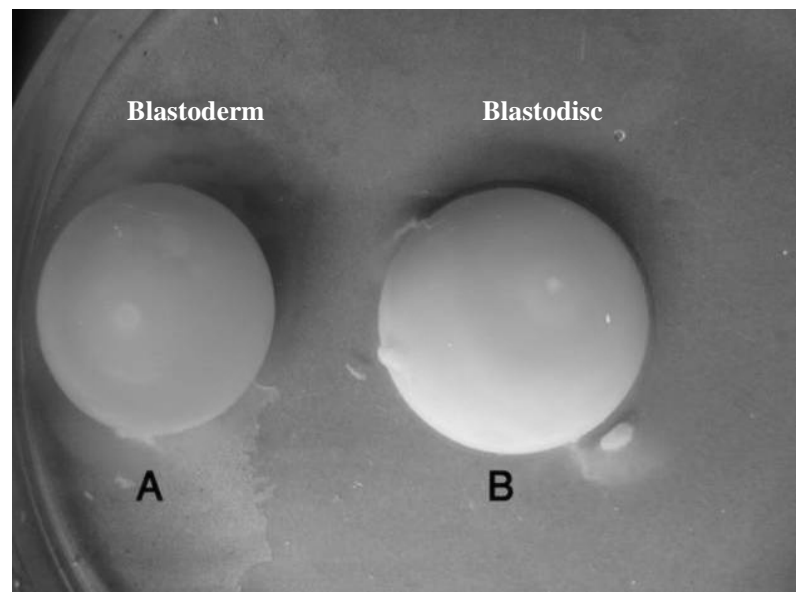

Fig. 6. A fertilized (A) and an unfertilized (B) egg of experimental hen.
Egg white (albumen): Egg white or albumen (Fig. 4. A) is the common name for the transparent cytoplasmic liquid contained within an egg.

Egg yolk: The egg yolk (Fig. 4. EY) is the part of an egg which serves as the food source for the developing embryo. Prior to fertilization the yolk together with the germinal disc is a single cell. It is one of the few singlecells that can be seen by the naked eye. The egg yolk is suspended in the egg white by chalazae. The shape of the egg yolk is round and contains germinal disc, and the entire structure is covered by transparent thin vitelline membrane (Rahman et al. 2007).

\section{Discussion}

The female reproductive system of chicken consists of the oviduct which is called as reproductive tract, and the ovary. After ovulation the ovum from the ovary, is engulfed by the funnel of infundibulum and transported along the tube and finally reached in the uterus. Before the egg is expelled out through the vent, the ova gradually mature in the oviduct by several maturity processes.

The oviduct of oviparous animals like chicken is an amazing organ and the morphology shows its several distinct portions playing different functions by producing each structural component of the laid egg, including the egg-white and eggshell. The ovary contains numerous follicles, some of which are with yolk material and some are devoid of it. All the ova start to mature one after another and grasped by the funnel of the infundibulum and only a small number of ova can reach maturity to produce an egg. Mature follicles containing fully matured yolk (Fig. 1) are ready for release into the oviduct. Empty or primary follicles with no yolk granules do not grow further. Beside these, there are discharged follicles, where the yolk has just been released; atretic follicles, those from which the yolk has been released some time ago.

Colour of the infundibular funnel is brownish and the next portion of it is reddish due to the presence of tubular glands and blood vessels. The infundibulummagnum junction is demarcated by a region from where comparatively less reddish magnum started. Inside the infundibulum several events occur, such as (i) the formation of inner portion of chalaziferous layer (Rahman et al. 2007, 2009b; Romanoff and Romanoff 1949; Burley and Vadehra 1989) or the outer layer of the vitelline membrane (Bakst and Howarth 1977), (ii) the storage of spermatozoa (Fujii and Tamura 1963; Bakst 1981; Bakst et al. 1994), (iii) fertilization (Olsen and Neher 1948; Bellairs et al. 1963; Howarth and 
Digby 1973; Okamura and Nishiyama 1978), and (iv) the formation of sperm associated bodies (SBs) (Sultana et al. 2004; Rabbani et al. 2006, 2007).

The ovulated ovum is grasped and literally swallowed by the infundibulum. This catching process is made easier by the left air sac which tightly encloses the ovary in the 'ovarian pocket' except caudally where the funnel opens. Nonetheless, not all ovulated oocytes are successfully captured. Some are lost into the body cavity where they are either harmlessly absorbed or become involved in egg peritonitis, which is known as internal laying. Penetration by spermatozoan occurs in the funnel. The inner portion of chalaziferous layer (Rahman et al. 2009b) and chalaza (Rahman et al. 2007) are formed from the infundibulum. Fertilization occurrs in the infundibulum, the first segment of the reproductive tract (Rabbani et al. 2006, 2007), which is the determinant factor that whether an ovum could be developed further and hatched or not.

The total time to form a new egg is 25 hours (Romanoff and Romanoff 1949). It takes about 1 hour to create the chalaza and chalaziferous layer in the infundibulum, 21/2 hours for developing the chalaza cord and egg white in magnum, and 20 hours for the secretion of shell itself in the uterus (Rahman et al. 2007, 2009a). In the isthmus egg stays for 60-70 min for the egg shell membrane and mammiliary core formation (Burley and Vadehra 1989). The present findings from decalcified shell membrane showed that the mammiliary cores were dissolved by EDTA and rendered structure which gave an unsmooth edge on the outer shell membrane (Fig. 5).

Most of the egg surface in the infundibulum is surrounded by the oviductal epithelium, except for the both ends where it is separated from the epithelium. The filaments (chalazae) originated from the chalaza materials at both presumptive ends, become extruded because of the absence of any physical force to push them inward (Rahman et al. 2007). The function of chalazae is to keep the yolk hanging in the egg (Burley and Vadehra 1989).

The third section of the oviduct the isthmus is smaller in length $(15 \mathrm{~cm})$ and the inner and outer two shell membranes of egg are added here. The developing egg remains here covered by the egg membranes for a short time comparing with that of infundibulum, magnum or uterus. From the lower red portion of isthmus calcification of egg starts. The calcium is secreted from the hen's bones and that might be a cause for their pneumatic bones in body.
The pouch like uterus is the expandable part of the oviduct which performs functions like: (i) production of the egg shell, (ii) the egg remains here for quite a long time, (iii) during the first 7 to 9 hours, secretory cells accumulate the watery substances from selective uterine glands and earlier to 21 hour egg shell formation occurs, (iv) in next 4 hours formation of cuticle provides the completion of chalaza and embryonic axis is formed (Rahman et al. 2007, 2009a; Rahman and Yoshizaki 2012), (v) deposition of pigment (if any) is done by the shell gland. Protoporphyrin tends to give brownish shell colours and the biliverdins blues and greens, and most white egg shells contain small amounts of pigment (Burley and Vadehra 1989). The shell contains pores to allow for transportation of oxygen for the embryo. An egg is surrounded by a thin, hard shell which helps the egg retaining its water and keep out the bacteria (Burley and Vadehra 1989).

The bloom or cuticle is added to the egg in the vagina prior to oviposition (Cited in Ferdous et al. 2011) but it has been proved that the cuticle is formed in the uterus during the second half of uterine period (Rahman et al. 2009a). Near the junction of the vagina and the shell gland, there present sperm host glands, also termed as sperm storage tubule (SST), where sperms can be stored for quite a long time (10 days to 2 weeks). The sperms are released during the egg passing from uterus to outside of body with the help of progesterone hormone (Ito et al. 2011). When an egg is laid, some of these sperm can be squeezed out of the glands into the oviduct so that they can migrate further up to the oviduct to fertilize an ovum. Within minutes after insemination, sperm reach the infundibulum, but they disappear within 24 hours and reappear in the lumen in small numbers at the time of each subsequent ovulation.

The soft cuticle covered the eggshell as foamy layer giving smoothness of egg for rotation during the stay in uterus (Rahman et al. 2009a). The cuticle is composed of two layers, a mineralized inner layer and an organic outer layer (Dennis et al. 1996). The cuticle also helps the egg keeping out bacteria and dust from the infestation.

Egg white is a source of complete protein and it contains all the amino acids required by the body for protein synthesis (Lewis et al. 1950). The egg white proteins are conalbumin, ovomucoid, ovomucin, lysozyme, globulins, flavoprotein, ovoglycoprotein, ovomacroglobulin, ovoinhibitor, avidin and cystatin (Li-Chan et al. 1995; Powrie 1973). It is used for nourishing the embryo during incubation and as high protein for human consumption. 
Vitelline membranes are of a meshwork of protein and glycoprotein fibers connecting each other by VMO-I and VMO-II (Rahman et al. 2009b). The inner layer is produced in the ovary whereas the outer layer is produced in the upper portion of the oviduct (Bain and Hall 1969) but Rahman et al. (2007, 2009b) reported that the outer layer of vitelline membrane is the inner portion of chalaziferous layer is produced in the posterior portion of the infundibulum. This structure keeps eggs free from polyspermy (Rabbani et al. 2006, 2007). The yolk of hen egg contains vitamins $A, D, E$ and $K$ as well as folic acid, pantothenic acid and zinc (ENC 2004).

The germinal disc (Fig. 4; G) is a small disc in the cytoplasm containing the nucleus can be seen on the surface of the yolk of a freshly laid egg as a circular, opaque white spot. It remains at the broader side of the egg because the ovum is never at the center of the overall structure; it is always closer to the blunt end (Rahman et al. 2007). When an egg is fertilized by a rooster, the blastodisc known as the blastoderm, which is the first stage of embryonic development. When incubated under particular temperatures and humidity levels for 21 days, these cells will develop into a chick. The blastoderm is characterized by the appearance of regular, concentric circles (Fig. 6 A). During incubation, the egg provides complete food for the developing embryo to be a chick.

In the fertilized egg (Fig. 4, G and Fig. 6. A.), between the blastoderm and the yolk there is a space called the subgerminal cavity which is created when the blastoderm absorb fluid from the albumin and present in between the blastodisc and the yolk. The deep cells in the centre of the blastoderm are leaving behind a one-cell-thick inner dot like structure the area pellucida and an outer ring like structure the area opaca. Between the area pellucida and the area opaca there is a thin layer of cells called the marginal zone. In case of unfertilized egg, there is a germinal disc on the surface of the yolk as an irregular white spot. Primordial germ cells (PGCs) are progenitor cells of ova and spermatozoa. PGCs originate from the epiblast of the central part of the area pellucida and translocate to the hypoblast at stages XI-XIV (Eyal- Giladi and Kochav 1976), and then carried anteriorly to the germinal crescent region, and finally migrate via the blood vascular system to the germinal ridges (undifferentiated gonads) where they differentiate into oogonia in female embryos and spermatogonia in male embryos (Kuwana, 1993).

Usually eggs are laid normally but sometimes there are some abnormalities and these could be resulted from various causes. Occasionally, a hen can produce double-yolked eggs which are not suitable for hatching. Yolkless eggs are usually formed when a bit of tissue is sloughed off the ovary or oviduct. This tissue stimulates the secreting glands of the different parts of the oviduct and a yolkless egg results (Jacob et al. 2011).

Blood spots are normally found on or around the yolk. The main cause is a small break in one of the tiny blood vessels around the yolk when it is ovulated. Occasionally an egg will be laid without a shell. The shell membranes are formed on the yolk and egg white, sometime somehow missing the "shell mechanism" and the shell was not deposited and it is resulting from a deficiency of calcium, phosphorus and/or vitamin D (Burley and Vadehra, 1989).

When faced with physical and/or microbial aggression, the egg has two major defensive mechanisms-a chemical protection system composed of yolk, albumen and eggshell matrix proteins that provide antimicrobial protection and the intact eggshell that acts as a physical barrier to protect against bacterial invasion (Mine and Kovacs-Nolan, 2006). The avian embryo is a higher vertebrate with the advantage of an extra uterine development and therefore it could be manipulated and observed the entire embryonic development by using eggs with a window in the egg shell or even egg shell free cultures (Borwornpinyo et al. 2005).

In the developed countries, the egg industry and technology is well established while in developing countries like Bangladesh eggs are mainly used as food. Adoption of egg technologies that utilize egg white and yolk for non-food uses will lead to produce more products from eggs. Egg albumin and yolk also have non-food uses in vaccine production, wine making, biotechnology, photography, painting, chemical and pharmaceutical industries.

\section{Conclusion}

This study contributes to our understanding of the morphology of reproductive system of hen from which ultimately eggs are produced. A working knowledge of the hen's reproductive system and the formation of egg assist the farmer to maximize egg production and quality, ensuring the supply of maximum animal protein source with minimum cost to the nation. Fertilized egg provides food for developing embryo during incubation whereas unfertilized as protein source for human consumption. The anatomical structure also assures egg quality depending on its goodness of constituent parts which could be achieved from maintaining proper environmental conditions after oviposition. Thus, the information provided here could be helpful to poultry anatomists, breeders, farmers and ordinary peoples. 


\section{References}

Ahmed S and Haque QME 1990. Avalable Feed Resources for Small Scale Poultry and Duck Production. Proceedings of the International Workshop on Crop and Animal Farming System Research, Asian Farming System Nrt-work, Dhaka, Bangladesh.

Aho P 2001. The world's commercial chicken meat and egg industries. In: Commercial Chicken Meat and Egg Production. 5th edn. (eds. Bell, D. D. and Weaver W. D.). Pp: 3-17. Kluwer Academic Publishers, the Netherlands.

Back JF Bain JM Vadehra DV and Burley RW 1982. Proteins of the outer layer of the vitelline membrane of hen's eggs. Biochem Biophys Acta 705:12-19

Bain JM and Hall JM 1969. Observations on the development and structure of the vitelline vembrane of the hen's eggs: an electron microscope study. Australian Journal of Biological Science, 22: 653-665

Bakst MR 1981. Sperm recovery from oviducts of turkeys at known intervals after insemination and oviposition. J Reprod Fertil. 62:159-164

Bakst MR and Howarth B Jr. 1977. The fine structure of the hen's ovum at ovulation. Biol Reprod. 17: 361-369

Bakst MR Wishart G and Brillard JP 1994. Oviductal sperm selection, transport and storage in poultry. Poult Sci Rev. 5: 117-143

Bellairs R Harkness M and Harkness RD 1963. The vitelline membrane of the hen's egg: a chemical and electron Microscope study. Journal of Ultrastructure Research. 8: 349-359

Borwornpinyo S Brake J Mozdziak PE and Petitte JN 2005. Culture of chicken embryos in surrogate eggshells. Poult Sci. 84: 1477-1482

Burley RW and Vadehra DV 1989. The Avian Egg: Chemistry and Biology. John Wiley \& Sons. New York. Pp 1-472

Das SC Chowdhury SD Khatun MA Nishibori M Isobe $\mathrm{N}$ and $\mathrm{Y}$. Yoshimora 2008. Poultry production profile and expected future projection in Bangladesh. World's poultry science journal. 64: 98-118

Dennis JE Xiao SQ Agarwal M Fink DJ Heuer AH and Caplan AI 1996. Microstructure of matrix and mineral components of eggshells from White Leghorn chickens (Gallus gallus). J. Morphol. 228: 287-306

DLS 2000. Expansions and Activities. Department of Livestock Services. Dhaka. Bangladesh pp. 31

ENC (Egg Nutrition Center) 2004. Egg protein fact sheet. http://www.enconline.org/ factsheet/EggProtein.pdf.

Eyal-Giladi H and Kochav S 1976. From cleavage to primitive streak formation: A complementary normal table and a new look at the first stages of the development of the chick I. General morphology. Developmental Biology. 49: 321-337.

Ferdous KA Parvez MNH and Rahman MT 2011. Effects of age on growth and development of vagina in ISA Brown chickens. The Bangladesh Vet. 28(2): 75-79

Fujii S and Tamura T 1963. Location of sperms in the oviduct of the domestic fowl with special reference to storage of sperms in the vaginal gland. J Fac Fish Husb Hiroshima Univ. 5: 145-163

Hernández-Hernández A Gómez-Morales J Rodríguez-Navarro AB Gautron J Nys Y and García-Ruiz JM 2008. Identification of some active proteins in the process of hen eggshell formation. Crystal Growth Design. 8: 4330-4339.
Hincke MT Chien YC Gerstenfeld LC and McKee MD 2008. Colloidal-gold immunocytochemical localization of osteopontin in avian eggshell gland and eggshell. J Histochem Cytochem. 56: $467-476$

Howarth B and Digby ST 1973. Evidence for the penetration of the hen's ovum by a trypsin-like acrosomal enzyme. J Reprod Fertil. 33: 123-125

Hossain MS 1992. Comparative study on egg production and feed efficiency of different breeds of poultry under intensive and rural conditions in Bangladesh. Livestock Research for Rural Development, 4(3) http://www.lrrd.org/lrrd4/3/bangla1.htm.

Ito T Yoshizaki N Tokumoto $\mathrm{T}$ Ono $\mathrm{H}$ Yoshimura $\mathrm{T}$ Tsukada A Kansaku N and Sasanami T 2011. Progesterone is a spermreleasing factor from the sperm-storage tubules in birds. Endocrinol. 152 (10): 3952-3962

Iwasawa A Rahman MA Roy TK Moriyama A \& Yoshizaki N 2010. Morphological and histochemical changes in the uterus epithelium during eggshell formation in quail. J Poult Sci. 47: 183-189

Jacob J Pescatore T and Cantor A. 2011. Avian female reproductive system. University of Kentucky College of Agriculture, Lexington.http://www2.ca.uky.edu/smallflocks/Factsheets/ Anatomy_and_Physiology/Anatomy_Female_reproductive.pdf

Khan MR \& Roy PC 2003. Credit policy; disbursement and its impact on poultry industry in Bangladesh. Third International Poultry Show and Seminar Dhaka Bangladesh: 43-51

Kuwana T 1993. Migration of avian primordial germ cells toward the gonadal anlage. Development, Growth and Differentiation. 35: 237-243

Lewis JC Snell NS Hirschmann DJ and Conrat HF 1950. Amino acid composition of egg proteins. J. Biol. Chem. 185: 23-35

Li-Chan ECY Powrie WD and Nakai S 1995. The chemistry of eggs and egg products. In: Egg science and technology. Stadelman,W.J. and Cotterhill (eds.). The Haworth press Inc., New York.

Mine Y and Kovacs-Nolan J 2006. New insights in biologically active proteins and peptides derived from the hen egg. World's Poultry Sci. 62: 87-95

Okada I Maeda Y Hashiguchi T Hasanath MA Faruque MO and Majid MA 1988. Gene constitution of indigenous chicken in Bangladesh. Jap Poultry Sci. 25: 15-26

Okamura F and Nishiyama H 1978. The passage of spermatozoa through the vitelline membrane in the domestic fowl Gallus gallus. Cell Tissue Res. 188: 497-508

Olsen MW and Neher BH 1948. The site of fertilization in the domestic fowl. J Exp Zool. 109: 355-366

Powrie W 1973. Chemistry of eggs and egg products. In: In Egg Science and Technology. Stadelman, W. and Cotterill. O. (eds.). Connecticut: AVI publishing Co, Westport. 61-90

Rahman MA 2009. Studies on the mechanism of chalaza formation in quail eggs. PhD Thesis, United Graduate School of Agricultural Science, Gifu University, Japan. pp: 1-89

Rahman MA Moriyama A Iwasawa A and Yoshizaki N 2009a. Cuticle formation in quail eggs. Zool. Sci. 26(7): 496-499

Rahman MA Moriyama A Iwasawa A and Yoshizaki N 2009b. VMO-II mediates the binding of the chalaziferous layer with the vitelline membrane in quail eggs. J. Pout. Sci. 46: 240-248 
Rahman MA Baoyindeliger Iwasawa A and Yoshizaki N 2007. Mechanism of chalaza formation in quail eggs. Cell and Tissue Research. 330: 535-543

Rahman MA and Yoshizaki N 2012. The time of embryonic axis formation in quail eggs. Univ. j. zool. Rajshahi Univ. 31: 89-90

Rabbani MG Sasanami T Mori M and Yoshizaki N 2006. Sperm-egg interaction is mediated by a sperm-associated body in quail. Development, Growth \& Differentiation. 48 (1): 33-40

Rabbani MG Sasanami T Mori M and Yoshizaki N 2007. Characterization of the sperm-associated body and its role in the fertilization of the chicken Gallus domesticus. Development, Growth \& Differentiation. 49 (1): 39-48
Roenigk WP 1999. World poultry consumption. Poult. Sci. 78: 722-728

Romanoff AL and Romanoff AJ 1949. The avian egg. Wiley, New York.

Sultana F Mao KM and Yoshizaki N 2004. Possible involvement of sperm- associated body in the process of fertilization in quail. Zool Sci. 21: 851-858

Woodard AE and Mather FB 1964. The timing of ovulation, movement of the ovum through the oviduct, pigmentation and shell deposition in Japanese quail (Coturnix coturnix japonica). Poult Sci. 43: 1427-1432

Manuscript received on 10 August 2013 and revised on 21 November 2013 